\title{
Null modes effect in Rossby wave model
}

\author{
V. Goncharov ${ }^{1}$ and V. Pavlov ${ }^{2}$ \\ ${ }^{1}$ Institute of Atmospheric Physics, Russian Academy of Sciences, 109017 Moscow, Russia \\ ${ }^{2}$ UFR de Mathématiques Pures et Appliquées, Université de Lille 1, 59655 Villeneuve d'Ascq, France
}

Received: 8 July 2003 - Revised: 1 April 2004 - Accepted: 5 April 2004 - Published: 28 May 2004

\begin{abstract}
The problem of the null-modes existence and some particularities of their interaction with nonlinear vortex-wave-like structures is discussed. We show that the null-modes are fundamental elements of nonlinear wave fields. The conditions under which null-modes can manifest themselves are elucidated. The Rossby-Hasegawa-Mima (RHM) model is used for the illustration of features of nullmodes-waves interactions.
\end{abstract}

\section{Introduction}

The purpose of the paper is to consider features of wave interactions in inhomogeneous, anisotropic, nonlinear media in a case when proper values, $\omega_{\boldsymbol{k}}$, for interacting wave modes, $a_{\boldsymbol{k}}$, turn to zero in some domain of $\boldsymbol{k}$-space. We present a new approach to solving the problem based on the Hamiltonian formulation of fluid dynamics (see Appendix A). We analytically study a new class of structures that can exist in two-dimensional flows governed by equations similar to the Rossby-Hasegava-Mima (RHM) ones (Hasegava and Mima, 1977; Kadomtsev, 1965; Similon and Sudan, 1990; Krall and Trivelpiece, 1973; Petviashvili and Pohotelov, 1989). These structures, termed below null-modes, turn out to be an important element in the theory of wave interactions and provoke a variety of intriguing questions. One task is to understand what physical reality corresponds to the basic state (null-modes) of the wave system when the proper values, $\omega_{\boldsymbol{k}}$, for interacting wave modes, $a_{k}$, become zero in some domain of the $\boldsymbol{k}$-space. Another one is to explore how the presence of null-modes modifies the wave interaction process as compared to the analogous well-studied process in homogeneous media. Finally, how does one develop field variables for the proper functions evolving in such a situation? Answers to these questions are of fundamental interest because so far all

Correspondence to: V. Pavlov

(vadim.pavlov@univ-lille1.fr) modern theories of wave interactions have been formulated in terms of normal modes $a_{k}$.

Before proceeding further, let us remind the reader that the concept of interacting waves is extensively used in wave physics and provides explanations for numerous collective effects. Its essence is that wave interaction is effectively realized only when the so-called resonance conditions on phases (frequencies and wave vectors) of interacting waves are satisfied (see for example Zakharov et al., 1985 and references therein).

For instance, in hydrodynamical systems, as experiments show (see Kadomtsev, 1965; Petviashvili and Pohotelov, 1989, and References therein), stationary flows sometimes appear as a result of intense field fluctuations. In geophysics, it is well-known that stationary (zonal) flows in atmospheres of rotating planets always coexist with intense surrounding wave fields. Based on the analogies from other branches of wave physics, it was once natural to assume that one of the principal mechanisms for stationary flow generation is the nonlinear interactions of waves. However, in geophysical hydrodynamics, application of the traditional approach to concrete situations is sometimes accompanied by difficulties. For example, the attempt to explain the appearance of stationary flows in the framework of three-wave resonance interaction of waves governed by the Rossby equations has failed: it has been shown that such a three-wave mechanism of interactions cannot be responsible for generation of stationary flows (see Longuet-Higgins and Gill, 1969; Pedlosky, 1986).

This theoretical result has instigated the approach which is developed below.

Let us remind the reader a few important facts. There exist various versions of the so-called Hamilton approach (HA) to field systems (see Dirac, 1958; Bogolubov and Shirkov, 1959; Lundgreen, 1963; Seliger and Whitham, 1968; Bretherton, 1970; Zakharov et al., 1985; Holm et al., 1985; Abarbanel et al., 1986; Salom, 1988; Dubrovin and Novikov, 1989; Goncharov and Pavlov, 1993; Zakharov and Kuznezov, 1997, and references theirein). 
The canonical version of the HA uses the following basic assumptions: the system under consideration is described by a set of canonical variables, symbolically marked here by $p$ and $q$. Generally, more than one pair of variables exists. The Fourier transformation of field variables from $\boldsymbol{x}$ space into $\boldsymbol{k}$-space, $(p, q) \rightarrow\left(p_{k}^{*}, q_{k}\right)$ is achieved, in order to identify the spectral wave components. A dispersion relationship between the frequency and the wave vector of the components is given by $\omega=\omega_{\boldsymbol{k}}$. Normal variables, $a_{k}, a_{k}^{*}$, are introduced by a linear transformation from $(p, q)$ to $\left(a_{\boldsymbol{k}}, a_{\boldsymbol{k}}^{*}\right)$ as coefficients for developing $p, q$ over corresponding eigenfunctions, $f_{\boldsymbol{k}}(\boldsymbol{x})$. In a homogeneous medium, $f_{\boldsymbol{k}}(\boldsymbol{x}) \sim \exp i \boldsymbol{k} \cdot \boldsymbol{x}$. The Hamiltonian of the system is the full energy expressed in terms of canonical variables, $H[p, q]$. In terms of normal variables, the quadratic part of the Hamiltonian developed in a functional series with respect to field variables, $H[p, q]=H_{2}+H_{3}+\ldots$, is presented as $H_{2}=\int d \boldsymbol{k} \omega_{\boldsymbol{k}} a_{\boldsymbol{k}} a_{-\boldsymbol{k}}$ and equations for normal modes evolution have the form $\partial_{t} a_{k}=-i \delta H / \delta a_{k}^{*}$ and $\partial_{t} a_{k}^{*}=+i \delta H / \delta a_{k}$. Here, $a_{k}^{*}=a_{-k}, \delta H / \delta u$ is a functional derivative with respect to field variable $u$. One assumes that $0<H[p, q]<+\infty$, which means that there are not of field perturbations at infinity: a physical problem of wave interactions is always considered in terms of wave packets.

Difficulties do not arise when the frequency of a wave mode depends on the absolute value of the wave vector, $\omega_{\boldsymbol{k}}=\omega_{|\boldsymbol{k}|}$, i.e. when the medium is homogeneous and isotropic. In this case, the Hamiltonian $\mathrm{H}_{2}$ is positive and remains positive when transformation $\boldsymbol{k} \rightarrow-\boldsymbol{k}$ is made. Difficulties appear when the media is anisotropic and proper value $\omega_{\boldsymbol{k}}$ depends on any particular direction, for example, when $\omega_{\boldsymbol{k}} \sim k_{x}$. Such a dependence follows, for instance, from the linearized version of Eq. (6). Obviously, in this case, resulting frequency cannot be simply introduced into the expression for $\mathrm{H}_{2}$ because $\mathrm{H}_{2}$ becomes null in this case. There is also no support or a motivated physical reason for including the absolute value $\left|\omega_{\boldsymbol{k}}\right|$ into $\mathrm{H}_{2}$.

For this reason only, a detailed analysis of the problem is essential.

The paper aims to achieve several goals: (i) to formulate, in a brief and relatively complete form, the fundamentals of one of the Hamilton approach versions; (ii) to formulate the concept of normal modes when the Hamiltonian of a system is defined by an operator expression; (iii) to introduce the canonical variables; (iv) to apply the developed methodology to the enigmatic physical phenomenon. It should be noted that without complying with certain rules, the procedure for introducing canonical variables, and normal variables as a consequence, is not as elementary as it could be expected.

In this article we perform the study in terms of generalized potentials ("canonical" field variables) $p$ and $q$, when the transition from field variables (velocities components) $\left(v_{1}, v_{2}\right)$ to $(p, q)$ is carried out, on the one hand, without a decrease of phase space dimension and, on the other hand, complying with the gauge invariance of the theory (see Sect. 3).
The article is structured as follows: Sect. 2 specifies the basic model and establishes some general results. In Sect. 3, we discuss the canonical formulation of the Hamiltonian description for fluid dynamics of nonlinear, dispersive, nonhomogeneous media. The Hamiltonian formulation for a Rossby-Hasegawa-Mima-like wave model is also proposed. The crucial importance of the proper introduction of normal variables in such systems is highlighted in Sect. 4. We consider the RHM-model as an example to which the HA can be directly applied to obtain analytical results. In Sect. 5, we discuss a linear approximation and explain where the nullmodes hide. In Sect. 6, the features of three-wave interactions are analyzed. A nonlinear collision of three wave packets is considered in Sect. 7. The null-mode mechanism of flow generation is considered in Sect. 8 and in Sect. 9, where all obtained results are discussed. In summary, we have found that specific structures (null-modes) can exist in strongly nonlinear and anisotropic wave systems. These not evolving in time structures (in linear approximation with respect to fields perturbations) correspond to the basic physical state when the stream-function, $\Psi$, is zero. The structures cannot be uncovered when one works with linearized equations, and, in some sense, they may be considered as "sleeping" ones. However, their presence can be observed when nonlinear interactions are taken into account with specific resonant conditions. One goal of the work was to find the conditions when such structures could be brought to light, in order to experimentally observe a generation of null-modes (i.e. observe the phenomenon of generation of time-independent flows by interacting wave fields). The realization of these conditions is so difficult that without preliminary theoretical analysis the effect may neither be guessed, nor observed experimentally.

\section{Basic model}

We consider a two-dimensional fluid motion, typical examples of which are large scale horizontal motions in planet atmospheres, hydrodynamical motions of a strongly magnetized plasma, etc. Such motions are described in the framework of the two-dimensional model of an incompressible perfect fluid. The velocity field is characterized in such a situation by two components only, $\boldsymbol{v}=\left(v_{1}, v_{2}, 0\right)$. The suppression of the third velocity component, $v_{3}$, can be caused by several physical causes. The evolution equations in this case are traditionally formulated in terms of generalized vorticity $\boldsymbol{\Omega}$ which is introduced by the relation $\Omega=\operatorname{curl} \boldsymbol{v}$. For twodimensional motions, only one component of vorticity, $\Omega_{3}$, is not null. The condition of incompressibility, div $\boldsymbol{v} \equiv \partial_{i} v_{i}=0$, permits to introduce the stream-function, $\Psi$, via the relation $v_{i}=\epsilon_{i j} \partial_{j} \Psi$, where $i, j=1,2, \epsilon_{i j}$ is an antisymmetrical unit tensor, $\epsilon_{12}=1=-\epsilon_{21}, \epsilon_{11}=\epsilon_{22}=0$.

The evolution of two-dimensional vorticity-like systems are governed by the equation

$\partial_{t} \Omega=-(\boldsymbol{v} \cdot \nabla) \Omega$, 
which can be written as

$\partial_{t} \Omega=-\left(\partial_{2} \psi\right) \partial_{1} \Omega+\left(\partial_{1} \psi\right) \partial_{2} \Omega$

The right part of this equation can be rewritten in terms of Jacobian, $J[a, b]$, where the Jacobian is defined as $J[a, b]=\partial_{1} a \partial_{2} b-\partial_{2} a \partial_{1} b \equiv \epsilon_{i j} \partial_{i} a \partial_{j} b$. Equation (2) conserves the full energy of the fluid motion

$H=\frac{1}{2} \int d \boldsymbol{x} \rho \psi \Omega$.

We suppose that all field variables and their derivatives, i.e. all perturbations, vanish in infinity. Also, background stationary flows are presumed to be absent.

The generalized vorticity, $\Omega$, and the stream-function, $\psi$, are related in general case by the operator relationship

$\Omega=\hat{L} \Psi$

The specification of a concrete physical model is assured by a relation between the stream-function and vorticity.

So, for a two-dimensional Euler equation, the indicated relationship has the form $\Omega=-\Delta \psi$, i.e. $\hat{L} \psi \equiv-\Delta \psi$. Here, $\Delta=\partial_{x}^{2}+\partial_{y}^{2}$ the two-dimensional Laplacian. Below we will frequently use $x \equiv x_{1}, y \equiv x_{2}$.

There exist other models (for example, the models of so-called "screened" vortices) with the operator $\hat{L}=-\left(\Delta-1 / a^{2}\right)$, where $a$ is some space scale defined by the choice of the model. Such models are largely used to describe (i) different plasma motions based on the HasegawaMima equation ((Hasegava and Mima, 1977; Similon and Sudan, 1990)), (ii) axial electronic vortices (Krall and Trivelpiece, 1973), (iii) the quasi-geostrophic barotropic motions (see below), etc. There exist even more complex examples (Gruzinov, 1992).

Let us provide as an illustration one visual example. In the geo-astrophysical context, it is typical to use a quasigeostrophic barotropic model defined by the relationship

$\Omega=\hat{L} \Psi+\beta y, \quad \beta=\frac{2 \omega_{0}}{a} \cos \vartheta_{0}$.

Here, $\hat{L}$ can be given by $\hat{L}=-\left(\Delta-1 / a^{2}\right)$. The term linear in $y$ accounts, in the first approximation, for the sphericity effect $(\beta$-effect), i.e. the variation of the Coriolis force with latitude $\vartheta$. Equation (5) is used in analyzing large-scale motions in an atmosphere considered as a thin layer of a fluid rotating with the angular velocity $\omega_{0}$. Let us note that Eq. (2) describes in this context two-dimensional fluid motions even if $\hat{L}$ and $\Psi$ depend on the vertical coordinate $z$.

Equations (2-4) form the closed system of equations for $\Omega, \Psi$.

Below, we consider the simplest case of two-dimensional motions where the stationary density, $\rho_{s}=\rho_{0}$, is independent on the vertical coordinate $z$. In the integral $H$, after integrating on $z, z$-dependence vanishes. Choosing mass, distance, time scales for which $\rho_{0}=1, a=1, \beta=1$ and scaling the full energy to obtain a dimensionless expression for $H$, the dimensionless stream-function, $\psi$, will be governed by the dimensionless evolution equation in the plane $x, y$

$\partial_{t}(\Delta-1) \psi+\partial_{x} \psi+J[\psi, \Delta \psi]=0$.

where the vorticity and the stream-function are related by the relationship

$\Omega=-(\Delta-1) \psi+y$.

The full energy of the fluid is then transformed into

$H \rightarrow H=\frac{1}{2} \int d \boldsymbol{x} \psi \Omega=-\frac{1}{2} \int d \boldsymbol{x} \psi(\Delta-1) \psi$.

\section{Hamiltonian approach}

The model governed by Eqs. (2-4) (see also Eqs. (6-8)) illustrates the Hamiltonian system with a finite number of fields and with a continual number of degrees of freedom characterized by the index $\boldsymbol{x}$. For this reason, functional derivatives with respect to field variables are used. To address the issue we use the version of the HA given in Goncharov and Pavlov (1984, 1993, 1997, 1998, 2001, 2002) and Pavlov et al. (2001). Information on supplementary bibliography can be found in Zakharov et al. (1985).

We will call the system a Hamiltonian one if it evolves according to

$\partial_{t} u_{i}=\left\{u_{i}, H\right\}=\int d \boldsymbol{x}^{\prime}\left\{u_{i}, u_{j}^{\prime}\right\} \frac{\delta H}{\delta u_{j}\left(\boldsymbol{x}^{\prime}\right)}$.

Here, the Hamiltonian of the system, $H$, is the quantityenergy-functionally dependent on the fields, $u_{i}$, the operator $\delta / \delta u$ is the operator of the functional derivative. The derivatives of dynamical variables, $F[u]$, are calculated by using the relation $\delta u_{i}(\boldsymbol{x}) / \delta u_{j}\left(\boldsymbol{x}^{\prime}\right)=\delta_{i j} \delta\left(\boldsymbol{x}-\boldsymbol{x}^{\prime}\right)$.

The Hamiltonian structure of the system described by Eq. (9) includes the Hamiltonian given by the total energy, $H$, and the functional Poisson bracket $\{.,$.$\} . This bracket$ is antisymmetric, bilinear, and satisfies the functional Jacobi identity presented symbolically in the following form

$\{A,\{B, C\}\}+\{B,\{C, A\}\}+\{C,\{A, B\}\}=0$,

Condition Eq. (10) must be obligatory satisfied for Hamiltonian systems.

Conservation of energy follows from the given formulation of governing equations, since $-\partial_{t} H=\{H, H\}=0$.

\subsection{Noncanonical formulation}

Systems which evolve according to Eqs. (2-4), give an example of such Hamiltonian systems. In fact, Eqs. (2-4) can be rewritten as

$\partial_{t} \Omega=\{\Omega, H\}=\int d x^{\prime}\left\{\Omega, \Omega^{\prime}\right\} \frac{\delta H}{\delta \Omega^{\prime}}$. 
Let us show how the bracket $\left\{\Omega, \Omega^{\prime}\right\}$ is calculated for concrete situations. Using expressions (8) and (11), we obtain that

$$
\{\Omega, H\}=\int d \boldsymbol{x}^{\prime} \psi^{\prime}\left\{\Omega, \Omega^{\prime}\right\}
$$

Here and further, the prime indicates that a field variable depends on the primed two-dimensional space coordinate $\boldsymbol{x}^{\prime}=\left(x^{\prime}, y^{\prime}\right)$. On the other hand, using Eq. (2), we can exclude $\partial_{t} \Omega$ from Eq. (11). The following step is to introduce the Jacobian under the integration operator where the properties of delta function are used. The regrouping of the integrand terms (all field variables vanish at infinity) yields

$$
\int d \boldsymbol{x}^{\prime} \psi^{\prime}\left[J\left(\delta\left(\boldsymbol{x}-\boldsymbol{x}^{\prime}\right), \Omega\right)-\left\{\Omega, \Omega^{\prime}\right\}\right]=0 .
$$

The expression in the square brackets is zero because Eq. (13) is satisfied for any $\psi$. Therefore, the Poisson bracket for the potential vorticity $\Omega$ can be written as

$$
\left\{\Omega, \Omega^{\prime}\right\}=J\left(\delta\left(\boldsymbol{x}-\boldsymbol{x}^{\prime}\right), \Omega\right) \text {. }
$$

This expression satisfies the Jacobi requirement, Eq. (10), which are necessary for Poisson brackets. The result, Eq. (14), can be obtained also by the direct calculation of the bracket on the canonical basis.

However, the formulation in terms of vorticity is sometimes uncomfortable because the Poisson bracket for such systems is functionally dependent of the field variables. By this reason, the canonical formulation of the problem is sometimes more preferable.

\subsection{Canonical formulation of RHM-like wave model}

The initial physical system is described by evolution equations of Euler which are formulated in terms of "measurable" physical variables, i.e. in terms of velocities $v_{1}, v_{2}$. Below, we will describe the same system in terms of canonical variables $p, q$ for which the functional Poisson brackets have the form : $\left\{p, q^{\prime}\right\}=\delta\left(\boldsymbol{x}-\boldsymbol{x}^{\prime}\right),\left\{p, p^{\prime}\right\}=\left\{q, q^{\prime}\right\}=0$, i.e. when the corresponding Poisson's brackets for field variables are independent on field variables. Such a choice is presented as more "natural" because the transition $v_{1}, v_{2} \rightarrow p, q$ conserves the same number of field variables, i.e. the same dimension of the phase space. In this case, an evolution of the system based on Eq. (6) will be described by an alternative, canonical, form. It signifies that the canonical variables satisfy equations

$\partial_{t} q=\frac{\delta H}{\delta p}, \quad \partial_{t} p=-\frac{\delta H}{\delta q}$,

where the Hamiltonian $H$, the total energy expressed in terms of canonical field variables $p, q$, is given by

$H=-\frac{1}{2} \int d \boldsymbol{x} \psi(p, q)(\Delta-1) \psi(p, q)$,

and where $\psi$ is expressed in terms of $q, p$. Variables $q, p$ have the meaning of the generalized coordinate and momentum, respectively.
In this context, some comments on the subject must be made.

What is the essence of the proposal "to introduce canonical variables"? Obviously, one possible way is to express the field variables, for example $v_{i}$, in terms of generalized potentials, $p, q$, which satisfy some (canonical) conditions. Thus, the problem is reduced to a search of a functional dependence $v_{i}[p, q]$. The dependence and corresponding canonical variables of such a type are known as Clebsh representations. It was A. Clebsh (1859) who pioneered using of similar transformations for a hydrodynamical velocity in an incompressible fluid (see for example Lamb, 1932). For some (simplest) systems, the canonical variables (potentials) $q$ and $p$ for incompressible fluid are introduced by $v_{k}=\partial_{k} \Phi+p \partial_{k} q$ (a review of more complex situations is given in Goncharov and Pavlov (1993)). Constraint $\operatorname{div} \boldsymbol{v}=0$ signifies that the potential $\Phi$ can be eliminated from consideration because for an incompressible fluid we have $\Delta \Phi=-\partial_{i}\left(p \partial_{i} q\right)$. The vorticity is defined via the Clebsh potential by the expression $\Omega_{i}=\varepsilon_{i j k} \partial_{j} p \partial_{k} q$. For two-dimensional flows, $i=3$ and the tensor Levy-Civita $\varepsilon_{i j k}$ becomes $\varepsilon_{3 j k} \equiv \varepsilon_{j k}$. However, it is clear that there is some functional arbitrary rule for choosing of a canonical basis $(p, q)$ for the given physical field $v_{i}$. To remove this arbitrary rule, one postulates that the theory must possess the gauge invariance. The criterion for removing the arbitrary rule is a possibility of the existence of such canonical transformations under which all physical (measured) quantities of the theory are kept invariant. This principle is called the principle of gauge invariance, generates in turn some specific laws of conservation which eliminate an over-determination of the system. The existence of such laws means, from a geometrical standpoint, that an evolution of the system is realized on some surface in the symplectic space $p, q$ which is fixed by indicated lows of conservation.

One can show that the relationship between the velocity components $v_{1}, v_{2}$ and the potentials $p, q$ is given by the sufficiently cumbersome nonlinear differential-integral expression

$$
(\Delta-1) v_{k}=\varepsilon_{k n} \partial_{n}\left(\varepsilon_{i j} \partial_{i} q \partial_{j} p+\partial_{2} p+x_{2} \partial_{1} q\right),
$$

Let us demonstrate this formula for a single pair of canonical variables $p, q$, i.e. for plane flows when only one pair of potentials is used. In this case, we have:

$\Omega=J(p, q)=\varepsilon_{i j} \partial_{i} p \partial_{j} q$.

The variables $q, p$ composing the canonical basis have meaning of the canonical coordinate and the canonical momentum, respectively. Thus, evolution equations formulated in terms of canonical variables $p, q$ are given by

$\partial_{t} p=-\frac{\delta H}{\delta q}, \quad \partial_{t} q=\frac{\delta H}{\delta p}$.

After calculating the functional derivatives of $H$, one finds that

$\partial_{t} p=J(p, \psi), \quad \partial_{t} q=J(q, \psi)$. 
Let us consider a quasi-geostrophic model (see Sect. II) with $\beta \neq 0$ The basic dimensionless relationship between the stream-function and the generalized vorticity is given by

$\Omega=\hat{L} \psi+x_{2}$,

which is expressed by Eq. (18) too. For this reason, we have the expression which gives a relationship between the stream-function and canonical variables $p$ and $q$

$\hat{L} \psi+x_{2}=J(q, p)$.

Let us suppose now that a state of dynamical equilibrium exists. The equilibrium values of variables corresponding to this state are marked by index $s$. Let us suppose that the background flow is absent, i.e. $\psi_{s}=0$. However, even if $\psi_{s}=0$, the problem arises for determining the nontrivial equilibrium values for the canonical variables (see for example Goncharov, 1984). So, we can find from Eqs. (20) and (18) that the stationary state, $\psi_{s}=0$, is determined by

$\partial_{t} p_{s}=0, \quad \partial_{t} q_{s}=0$,

$x_{2}=J\left(p_{s}, q_{s}\right)$,

where Eq. (24) follows from the two definitions of the vorticity presented above. Evidently, the solutions of Eq. (23) are two time-independent functions which satisfy only one Eq. (24). For this reason, we can choose one of the potentials $p_{s}$ or $q_{s}$ arbitrarily. Choosing the function $p_{s}$ as

$p_{s}=x_{1}$,

we obtain

$q_{s}=\frac{1}{2} x_{2}^{2}$

All physical ("measurables") fields do not change in this case.

Now, it is convenient to introduce new canonical variables

$p^{\prime}=p-p_{s}, \quad q^{\prime}=q-q_{s}$,

which in the equilibrium regime satisfy to the condition $p_{s}^{\prime}=q_{s}^{\prime}=0$.

In terms of perturbations $p^{\prime}, q^{\prime}$, relationship (18) and (20) are written as

$\hat{L} \psi=x_{2} \partial_{1} q^{\prime}+\partial_{2} p^{\prime}+J\left(q^{\prime}, p^{\prime}\right)$,

$\partial_{t} q^{\prime}=\frac{\delta H}{\delta p^{\prime}}=J\left(q^{\prime}+x_{1}, \psi\right)$,

$\partial_{t} p^{\prime}=-\frac{\delta H}{\delta q^{\prime}}=J\left(\frac{1}{2} x_{2}^{2}+p^{\prime}, \psi\right)$.

Omitting the prime, one sees that Eq. (28) is transformed into Eq. (17) if we apply to Eq. (28) operator $\varepsilon_{i j} \partial_{j}$.

Let us move now into $\boldsymbol{k}$-space, using the symmetric Fourier transforms defined by the formula

$Z_{\boldsymbol{k}}=\int \frac{d \boldsymbol{x}}{2 \pi} Z(\boldsymbol{x}) \exp (-i \boldsymbol{k} \cdot \boldsymbol{x})$.
The goal of such a transformation is, first, to obtain the dispersion relationship for wave components and, second, to apply the results of the theory to interacting wave components with fixed wave vectors.

In this case, according to Eqs. (15-17), the Fourier components evolution is governed by nonlinear equations

$\partial_{t} q_{\boldsymbol{k}}=\frac{\delta H}{\delta p_{\boldsymbol{k}}^{*}}, \quad \partial_{t} p_{\boldsymbol{k}}^{*}=-\frac{\delta H}{\delta q_{\boldsymbol{k}}}$,

The asterisk $\left(^{*}\right)$ denotes the complex conjugate.

Concerning a possibility of the Fourier transformations, we have supposed that the background flows are absent and all field variables (perturbations) vanish rapidly in infinity, i.e. even $|\boldsymbol{x}| \psi \rightarrow 0$ when $|\boldsymbol{x}| \rightarrow \infty$. Such a supposition is largely used in the framework of traditional approaches in physics when models of wave packets are considered. In this case, all physical operators are transformed according to $\hat{A}(\boldsymbol{x}, \nabla) \rightarrow \hat{A}_{\boldsymbol{k}}\left(-i \nabla_{\boldsymbol{k}}, i \boldsymbol{k}\right)$. Multiplying Eq. (17) by the exponent, integrating over the space coordinate and neglecting terms in infinity, we can find that the stream-function component $\psi_{\boldsymbol{k}}$ and the canonical variables' components $q_{\boldsymbol{k}}, p_{\boldsymbol{k}}$ are related by the relationship

$$
\begin{aligned}
& \psi_{\boldsymbol{k}}=-\frac{1}{\boldsymbol{k}^{2}+1}\left[i \sigma p_{\boldsymbol{k}}-\kappa \frac{\partial q_{\boldsymbol{k}}}{\partial \sigma}\right. \\
&\left.+\int \frac{d \boldsymbol{k}_{1} d \boldsymbol{k}_{2}}{2 \pi} q_{\boldsymbol{k}_{1}} p_{\boldsymbol{k}_{2}}\left(\sigma_{1} \kappa_{2}-\sigma_{2} \kappa_{1}\right) \delta\left(\boldsymbol{k}_{1}+\boldsymbol{k}_{2}-\boldsymbol{k}\right)\right] .
\end{aligned}
$$

Here, $\kappa$ and $\sigma$ are longitudinal and transversal components of the wave vector $\boldsymbol{k}$, i.e. $\boldsymbol{k}=(\kappa, \sigma)$. The operators act on all variables which are positioned to the right of them.

The Hamiltonian is given by the simple expression

$H[p, q]=\frac{1}{2} \int d \boldsymbol{k}\left(\boldsymbol{k}^{2}+1\right)\left|\psi_{\boldsymbol{k}}\right|^{2}$,

where the function $\psi_{k}$ has to be expressed in terms of the canonical variables $q_{\boldsymbol{k}}, p_{\boldsymbol{k}}$.

The Hamiltonian Eq. (33) is the functional polynômial of the fourth order with respect to field (canonical) variables $q_{\boldsymbol{k}}$, $p_{k}$. It can be written as

$H[p, q]=H_{2}[p, q]+H_{3}[p, q]+H_{4}[p, q]$,

where $H_{2}[p, q]$ describe linear effects, and $H_{3,4}[p, q]$ nonlinear effects of interacting perturbations into the system.

\section{Normal variables}

The problem of introducing normal variables is not trivial because operator combinations appear in Eq. (32). Similar combinations will appear in $H_{2}[p, q]$.

Let us write Eqs. (31) in a matrix form

$\partial_{t} \boldsymbol{u}_{\boldsymbol{k}}=-i \hat{J} \frac{\delta H}{\delta \boldsymbol{u}_{\boldsymbol{k}}^{+}}$, 
where $\left({ }^{+}\right)$denotes the Hermitian conjugate. The column vectors $\boldsymbol{u}_{\boldsymbol{k}}$ and so-called symplectic matrix $\hat{J}$ are arranged as

$\boldsymbol{u}=\left(\begin{array}{c}p_{\boldsymbol{k}} \\ q_{\boldsymbol{k}}\end{array}\right), \quad \hat{J}=\left(\begin{array}{cc}0 & -i \\ i & 0\end{array}\right)$.

The Poisson brackets can be written as

$\left\{\boldsymbol{u}_{\boldsymbol{k}}, \boldsymbol{u}_{\boldsymbol{k}^{\prime}}^{+}\right\}=-i \hat{J} \delta\left(\boldsymbol{k}-\boldsymbol{k}^{\prime}\right)$.

Let us consider small field perturbations relative to basic unperturbed state. In this case, the leading term into the Hamiltonian $H$ is the quadratic term $H_{2}[p, q]$ with respect to canonical variables $p$ and $q$. This approximation corresponds to the linear approximation for evolution equations. For $H_{2}[p, q]$, we can write the following representation

$H_{2}=\frac{1}{2} \int d \boldsymbol{k} \boldsymbol{u}_{\boldsymbol{k}}^{+} \hat{H}_{\boldsymbol{k}} \boldsymbol{u}_{\boldsymbol{k}}$

The matrix operator $\hat{H}_{k}$ have the following structure

$\hat{H}_{k}=\left(\begin{array}{cc}A_{k} & \hat{B}_{k} \\ \hat{B}_{k}^{+} & \hat{C}_{k}\end{array}\right)$,

where matrix element $A_{k}$ is a function of $\sigma$ and $\kappa$, while elements $\hat{B}_{k}$ and $\hat{C}_{k}$ are operators. These elements are expressed by

$A_{k}=\frac{\sigma^{2}}{\boldsymbol{k}^{2}+1}$,

$\hat{B}_{\boldsymbol{k}}=\frac{i \sigma \kappa}{\boldsymbol{k}^{2}+1} \frac{\partial}{\partial \sigma}, \quad \hat{C}_{\boldsymbol{k}}=-\frac{\partial}{\partial \sigma} \frac{\kappa^{2}}{\boldsymbol{k}^{2}+1} \frac{\partial}{\partial \sigma}$.

By definition (see below), normal variables $a_{k}(t)$ are introduced as coefficients in decomposition

$\boldsymbol{u}_{\boldsymbol{k}}=\hat{\boldsymbol{f}}_{\boldsymbol{k}} a_{\boldsymbol{k}}+\hat{\boldsymbol{f}}_{-\boldsymbol{k}}^{*} a_{-k}^{*}$,

where $f_{k}, f_{-k}^{*}$ are vector-column eigenfunctions.

In terms of normal variables, the two equations of evolution Eq. (35) are transformed into

$\partial_{t} a_{k}=-i \frac{\delta H}{\delta a_{k}^{*}}, \quad \partial_{t} a_{k}^{*}=i \frac{\delta H}{\delta a_{k}}$.

In linear approximation, $H \rightarrow H_{2}$. The Hermitian matrix operator $\hat{H}_{\boldsymbol{k}}$ at Eq. (38) has a typical structure Eq. (39). Considering the fact that $\hat{H}$ is Hermitian, $H_{2}$ is real, and $\boldsymbol{u}_{\boldsymbol{k}}=\boldsymbol{u}_{-\boldsymbol{k}}^{*}$, one sees that

$\hat{H}_{k}=\hat{H}_{k}^{+}=\hat{H}_{-\boldsymbol{k}}^{*}$.

It means that

$\hat{A}_{k}=\hat{A}_{k}^{+}=\hat{A}_{-k}^{*}, \quad \hat{C}_{k}=\hat{C}_{k}^{+}=\hat{C}_{-k}^{*}, \quad \hat{B}_{k}=\hat{B}_{-k}^{*}$.

Substituting Eq. (38) into the Hamiltonian Eqs. (35), we obtain the linear equation

$$
\left(\hat{J} \partial_{t}+i \hat{H}_{k}\right) \boldsymbol{u}_{\boldsymbol{k}}=0 .
$$

To formulate the problem on eigenfunctions and eigenvalues, we must substitute $\boldsymbol{u}_{\boldsymbol{k}}=\hat{\boldsymbol{f}}_{\boldsymbol{k}} \exp (-i \omega t)$ in Eq. (45), assuming that, in general, the eigenvectors $\hat{f}_{k}$ are operators non-commuting with the eigenvalues $\omega=\omega_{\boldsymbol{k}}$. In doing so, we obtain

$\hat{H}_{k} \hat{\boldsymbol{f}}_{\boldsymbol{k}}-\hat{J} \hat{\boldsymbol{f}}_{\boldsymbol{k}} \omega_{\boldsymbol{k}}=0$,

It should be noted that as far as $\hat{\boldsymbol{f}}_{\boldsymbol{k}} \omega_{\boldsymbol{k}} \neq \omega_{\boldsymbol{k}} \hat{\boldsymbol{f}}_{\boldsymbol{k}}$, this problem cannot be reformulated in the form

$\left(\hat{H}_{k}-\omega_{k} \hat{J}\right) \hat{f}_{k}=0$

which is traditional for expressions with no operators.

Using the properties $\hat{H}_{k}=\hat{H}_{-k}^{*}, \hat{J}=-\hat{J}^{*}$, we can derive from Eq. (46) the dual linear problem

$\hat{H}_{\boldsymbol{k}} \hat{\boldsymbol{f}}_{-\boldsymbol{k}}^{*}+\hat{\boldsymbol{J}} \hat{\boldsymbol{f}}_{-\boldsymbol{k}}^{*} \omega_{-\boldsymbol{k}}=0$.

Comparing Eq. (46) with Eq. (47) shows that if $\omega_{\boldsymbol{k}}$ and $\hat{\boldsymbol{f}}_{\boldsymbol{k}}$ are an eigenvalue and an eigenvector then $-\omega_{-\boldsymbol{k}}$ and $\hat{\boldsymbol{f}}_{-\boldsymbol{k}}^{*}$ are too. Therefore, all the eigenvalues and the eigenvectors of problem Eq. (46) have dual nature and can be split into such pairs. In the simplest case when a model has only one wave branch, the problem Eq. (46) has a single pair of eigenvectors $\left(\hat{f}_{\boldsymbol{k}}, \hat{\boldsymbol{f}}_{-\boldsymbol{k}}^{*}\right)$ and a corresponding pair of eigenvalues $\left(\omega_{\boldsymbol{k}},-\omega_{-\boldsymbol{k}}\right)$.

In this case, according to general theory tenets (Goncharov and Pavlov, 1993), because matrices $\hat{H}_{k}$ and $\hat{J}$ are Hermitian and, in addition, $\hat{H}_{k}$ is positively determined, there are good grounds for believing that, on the one hand, the eigenvectors $\hat{\boldsymbol{f}}_{\boldsymbol{k}}$ and $\hat{\boldsymbol{f}}_{-\boldsymbol{k}}^{*}$ form the system of orthonormal functions subjected to the conditions

$\hat{\boldsymbol{f}}_{\boldsymbol{k}}^{+} \hat{\boldsymbol{J}} \hat{\boldsymbol{f}}_{\boldsymbol{k}}=1, \quad \hat{\boldsymbol{f}}_{\boldsymbol{k}}^{+} \hat{\boldsymbol{J}} \hat{\boldsymbol{f}}_{-\boldsymbol{k}}^{*}=0$,

on the other hand, all the eigenvalues are real, i.e. $\Im \omega_{k}=0$.

Thus, normal variables $a_{k}(t)$ are introduced as coefficients in decomposition of the column vector $\boldsymbol{u}_{\boldsymbol{k}}$ on the eigenvectors $\left(\hat{\boldsymbol{f}}_{\boldsymbol{k}}, \hat{\boldsymbol{f}}_{-\boldsymbol{k}}^{*}\right)$ by Eq. (42).

Using orthonormality property Eq. (48), from Eq. (42) it is easy to obtain the relations

$a_{k}=\hat{\boldsymbol{f}}_{\boldsymbol{k}}^{+} \hat{J} \boldsymbol{u}_{\boldsymbol{k}}=-\boldsymbol{u}_{-\boldsymbol{k}}^{+} \hat{J} \hat{\boldsymbol{f}}_{\boldsymbol{k}}^{*}$,

and next, by employing Eq. (37), to calculate

$\left\{a_{k}, a_{\boldsymbol{k}^{\prime}}^{*}\right\}=-i \delta\left(\boldsymbol{k}-\boldsymbol{k}^{\prime}\right), \quad\left\{a_{\boldsymbol{k}}, a_{\boldsymbol{k}^{\prime}}\right\}=0$.

On the basis Eq. (50), the matrix equation of motion Eq. (35) takes form

$\partial_{t} a_{k}=-i \frac{\delta H}{\delta a_{\boldsymbol{k}}^{*}}, \quad \partial_{t} a_{\boldsymbol{k}}^{*}=i \frac{\delta H}{\delta a_{\boldsymbol{k}}}$.

To find solutions for $\hat{\boldsymbol{f}}$ and $\omega_{\boldsymbol{k}}$ in an explicit form in what follows we assume for simplicity that matrix element $A_{k}$ is a usual function while matrix elements $\hat{B}_{k}$, and $\hat{C}_{k}$ are noncommuting operators. 
In this case one can show that according to Eq. (46) eigenvector $\hat{\boldsymbol{f}}_{\boldsymbol{k}}$ is given by

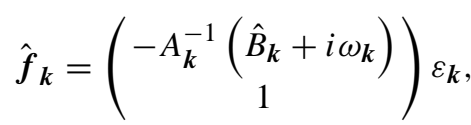

The eigenvalue $\omega_{\boldsymbol{k}}$ and the normalizing factor $\varepsilon_{\boldsymbol{k}}$, in turn, can be found from orthonormality conditions Eq. (48) which is convenient to reformulate, using Eq. (52), as

$\omega_{k}-\omega_{-k}=-i\left(A_{k} \hat{B}_{k}^{+} A_{k}^{-1}-\hat{B}_{k}\right)$,

$\omega_{-k} \omega_{k}=A_{k}\left(\hat{C}_{k}-\hat{B}_{k}^{+} A_{k}^{-1} \hat{B}_{k}\right)$,

$\left|\varepsilon_{\boldsymbol{k}}\right|^{2}=\left(\omega_{\boldsymbol{k}}+\omega_{-\boldsymbol{k}}\right)^{-1} A_{\boldsymbol{k}}$.

Here, $\omega_{\boldsymbol{k}}$ is the eigenvalue of the problem, $\varepsilon_{\boldsymbol{k}}$ is a normalization coefficient. To find the eigenvalue $\omega_{\boldsymbol{k}}$ and the normalizing factor $\varepsilon_{\boldsymbol{k}}$, we must resolve conditions Eqs. (53-55) with Eqs. (39) and (41), which lead to

$\omega_{k}-\omega_{-k}=-\frac{\kappa}{\boldsymbol{k}^{2}+1}$,

$\omega_{-k} \omega_{k}=0$,

$\left|\varepsilon_{\boldsymbol{k}}\right|^{2}=\frac{\sigma^{2}}{\left(\boldsymbol{k}^{2}+1\right)\left(\omega_{\boldsymbol{k}}+\omega_{-\boldsymbol{k}}\right)}$.

From Eqs. (56-58), we find

$\omega_{\boldsymbol{k}}=-\frac{\kappa \theta(-\kappa)}{\boldsymbol{k}^{2}+1}, \quad \varepsilon_{\boldsymbol{k}}=\sigma|\kappa|^{-1 / 2}$,

where $\theta(\kappa)$ is the Heaviside function: $\theta(\kappa)=1$ if $\kappa \geq 0$, and $\theta(\kappa)=0$ if $\kappa<0$. This law of wave dispersion is shown in Fig. 1. For $\kappa \leq 0$ it describes the well-known Rossby-like waves. The domain of $\kappa>0$ corresponds to so-called null modes.

Summing up this section, we list the basic relationships

$$
\begin{aligned}
q_{\boldsymbol{k}}=\sigma|\kappa|^{-1 / 2}\left(a_{\boldsymbol{k}}-a_{-\boldsymbol{k}}^{*}\right) & , \\
p_{\boldsymbol{k}}=-i|\kappa|^{1 / 2} \operatorname{sign}(\kappa) & {\left[\left(\frac{\partial}{\partial \sigma}+\frac{\theta(\kappa)}{\sigma}\right) a_{k}\right.} \\
& \left.-\left(\frac{\partial}{\partial \sigma}+\frac{\theta(-\kappa)}{\sigma}\right) a_{-k}^{*}\right] .
\end{aligned}
$$

The stream-function is defined via the normal variables by expression

$$
\begin{gathered}
\psi_{\boldsymbol{k}}=-|\kappa|^{-1 / 2}\left(\omega_{\boldsymbol{k}} a_{\boldsymbol{k}}+\omega_{-\boldsymbol{k}} a_{-\boldsymbol{k}}^{*}\right)-\frac{1}{2 \pi\left(\boldsymbol{k}^{2}+1\right)} \\
\times \int d \boldsymbol{k}_{1} d \boldsymbol{k}_{2} p_{\boldsymbol{k}_{2}} q_{\boldsymbol{k}_{1}}\left(\sigma_{1} \kappa_{2}-\sigma_{2} \kappa_{1}\right) \delta\left(\boldsymbol{k}-\boldsymbol{k}_{1}-\boldsymbol{k}_{2}\right) .
\end{gathered}
$$

where $p_{k}, q_{k}$ have to be expressed by Eq. (61).

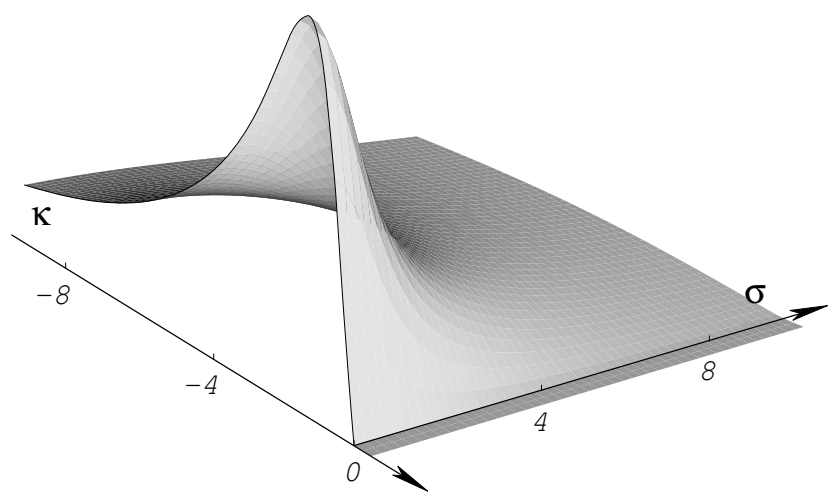

Fig. 1. A general dispersion law for the Rossby-like waves and null modes.

\section{Linear approximation}

In the linear approximation, when interaction of Rossby-like waves is ignored, the Hamiltonian takes form

$H_{2}=\int d \boldsymbol{k} \omega_{\boldsymbol{k}}\left|a_{\boldsymbol{k}}\right|^{2}>0$,

and, as earlier discussed, is positively defined since $\omega_{\boldsymbol{k}} \geq 0$ in accordance with Eq. (59). Due to the presence of $\theta(-\kappa)$ function in the dispersion law Eq. (59), null modes defined above as normal modes with positive components of $\kappa$, are eliminated in a linear approximation. Once initially established, these modes remain unchanged without any physical consequences or effects because, in accordance with Eq. (62), in a linear approximation we have

$$
\begin{aligned}
\psi(\boldsymbol{x}) & =-\frac{1}{2 \pi} \operatorname{Re} \int d \boldsymbol{k}|\kappa|^{-1 / 2} \omega_{\boldsymbol{k}} a_{\boldsymbol{k}} e^{i \boldsymbol{k} \boldsymbol{x}} \\
& \equiv \psi^{R}+\psi^{N M},
\end{aligned}
$$

and hence they make zero contribution to the streamfunction, $\psi^{N M}=0$, even if their amplitudes are distinct from zero.

As we shall see later, this is no longer the case in a nonlinear approximation.

The null-modes correspond to the state with $\Psi=0$. However, using the traditional consideration where governing equations are formulated in terms of a stream-function, it is very difficult to guess to which physical reality the states with $\Psi=0$ correspond. On the other hand, discovering that the stream-function $\Psi=\Psi[p, q]$ depends in reality on canonical field variables $(p, q)$ which arise from transition $\left(v_{1}, v_{2}\right) \rightarrow(p, q)$, it becomes clear that, even when $\Psi[p, q]$ is null, it does not automatically follow that $p$ and $q$ are also equal to zero. A physical reality, with $p, q \neq 0$ assuring $\Psi[p, q]=0$, can exist: the stream-function can turn to zero, for example, when field amplitudes (normal variables) or other characteristics of the wave field vanish in a certain domain of $\boldsymbol{k}$-space (null-modes). In this connection, it becomes clear that null-modes can in principle be initiated by some nonlinear wave interactions. 


\section{Three-wave interactions}

To find the types of wave interactions admissible by normal waves dispersion Eq. (59), we must consider resonance conditions which take null modes into account. Because $\omega_{\boldsymbol{k}} \geq 0$, and hence the so-called waves of the negative energy are absent, the main contribution at the first order of perturbation theory is made by the three-wave interactions corresponding to the resonance conditions

$\boldsymbol{k}-\boldsymbol{k}_{1}-\boldsymbol{k}_{2}=0, \quad \omega_{\boldsymbol{k}}-\omega_{\boldsymbol{k}_{1}}-\omega_{\boldsymbol{k}_{2}}=0$,

which describe decay processes $(0 \rightarrow 1+2)$ and inverse processes-merging $(1+2 \rightarrow 0)$.

Of special interest are the interactions between two Rossby-like waves and a null mode which arise whenever one of wave-vectors, for example $\boldsymbol{k}_{2}$, has a positive component $\kappa_{2}$ while components $\kappa$ and $\kappa_{1}$ are negative. In this case, wave-vectors of interacting Rossby-like modes form a locus where

$\omega_{k}=\omega_{k_{1}}$.

According to Eq. (66), the tips of vectors $\boldsymbol{k}$ and $\boldsymbol{k}_{1}$ must lie on the circle with radius $r=\sqrt{\omega_{\boldsymbol{k}}^{-2} / 4-1}$ and the center at point $\left(-\omega_{\boldsymbol{k}}^{-1} / 2,0\right)$ as graphically represented in Fig. 2 .

In order to proceed further, we must also know the Hamiltonian of the three-wave interactions which is responsible for resonance Eq. (65), and ignore all other feasible nonresonant terms which can be eliminated with the aid of the canonical transformation. Thus, we arrive at the Hamiltonian with a general structure

$$
\begin{array}{r}
\left.H_{3}=\frac{1}{2} \int d \boldsymbol{k} d \boldsymbol{k}_{1} d \boldsymbol{k}_{2}\left(a_{\boldsymbol{k}}^{*} a_{\boldsymbol{k}_{1}} a_{\boldsymbol{k}_{2}} V_{\boldsymbol{k}, \boldsymbol{k}_{\mathbf{1}}, \boldsymbol{k}_{2}}\right)+c . c\right) \\
\times \delta\left(\boldsymbol{k}-\boldsymbol{k}_{1}-\boldsymbol{k}_{2}\right),
\end{array}
$$

where function $V_{\boldsymbol{k}, \boldsymbol{k}_{1}, \boldsymbol{k}_{2}}$ is called the interaction factor or coupling coefficient. The coupling coefficient $V_{\boldsymbol{k}, \boldsymbol{k}_{1}, \boldsymbol{k}_{2}}$ can be obtained by expanding Eq. (33) into a functional power series of normal variables with subsequent collecting of terms proportionate to $a^{*} a a$ and $a a^{*} a^{*}$. Because our interest is only in resonance interactions, function $V_{\boldsymbol{k}, \boldsymbol{k}_{1}, \boldsymbol{k}_{2}}$ is defined only on the surface described by Eq. (65). Therefore, if we restrict ourselves to the three-wave interactions, terms proportional to $\left(\omega_{\boldsymbol{k}}-\omega_{\boldsymbol{k}_{1}}-\omega_{\boldsymbol{k}_{2}}\right)$ can be omitted in the computation of $V_{k, k_{1}, k_{2}}$.

After some algebra, we find

$$
\begin{aligned}
V_{\boldsymbol{k}, \boldsymbol{k}_{1}, \boldsymbol{k}_{2}}= & \frac{i\left(\sigma_{1} \kappa_{2}-\kappa_{1} \sigma_{2}\right)}{2 \pi \sqrt{\left|\kappa_{1} \kappa_{2}\right|}}\left\{\left(\omega_{\boldsymbol{k}_{1}} \sigma_{2}-\omega_{\boldsymbol{k}_{2}} \sigma_{1}\right)\right. \\
& \times\left(\frac{\kappa}{\sigma} \theta(\kappa)+\frac{\kappa_{1}}{\sigma_{1}} \theta\left(\kappa_{1}\right)+\frac{\kappa_{2}}{\sigma_{2}} \theta\left(\kappa_{2}\right)\right) \\
& +\left(\omega_{\boldsymbol{k}_{1}} \kappa_{2}-\omega_{\boldsymbol{k}_{2}} \kappa_{1}\right)-\left(\sigma_{1} \kappa_{2}-\kappa_{1} \sigma_{2}\right) \\
& \left.\times\left(\frac{\partial \omega_{\boldsymbol{k}}}{\partial \sigma}-\frac{\partial \omega_{\boldsymbol{k}_{1}}}{\partial \sigma_{1}}-\frac{\partial \omega_{\boldsymbol{k}_{2}}}{\partial \sigma_{2}}\right)\right\} .
\end{aligned}
$$

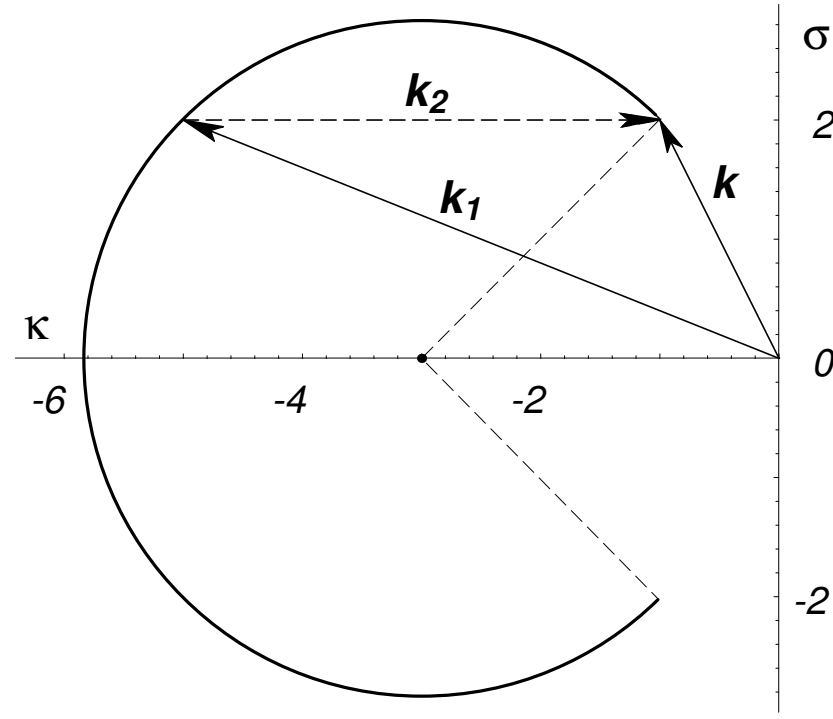

Fig. 2. Locus of the null-mode wave-vector tails merging with a Rossby-like mode. Dashed-line vector $\boldsymbol{k}_{2}$ denotes the null mode, solid-line vectors $\boldsymbol{k}$ and $\boldsymbol{k}_{1}$ denote the Rossby-like modes.

\section{Nonlinear collision of three wave packets}

We consider the interaction of three narrow-band wave packets whose typical wave-vectors $\boldsymbol{k}_{1}, \boldsymbol{k}_{2}, \boldsymbol{k}_{3}$ satisfy the resonance conditions

$\boldsymbol{k}_{1}=\boldsymbol{k}_{2}+\boldsymbol{k}_{3}, \quad \omega_{\boldsymbol{k}_{1}}=\omega_{\boldsymbol{k}_{2}}+\omega_{\boldsymbol{k}_{3}}$.

Let us assume that

$a(\boldsymbol{k})=a_{1}(\boldsymbol{k})+a_{2}(\boldsymbol{k})+a_{3}(\boldsymbol{k})$,

where each of $a_{n}$ is nonzero only if $\boldsymbol{q}=\boldsymbol{k}-\boldsymbol{k}_{n}$ are small. Fast dependence on $t$ and $\boldsymbol{x}$ for each wave packet is excluded with transformation

$c_{n}(\boldsymbol{q})=a_{n}\left(\boldsymbol{k}_{n}+\boldsymbol{q}\right) \exp i\left(\omega_{\boldsymbol{k}_{n}} t-\boldsymbol{k}_{n} \boldsymbol{x}\right)$.

Following the standard procedure (see, e.g. Zakharov and Kuznetsov, 1986), using new variables

$u_{n}(\boldsymbol{x})=(2 \pi)^{-1} \int d \boldsymbol{q} c_{n}(\boldsymbol{q}) e^{i \boldsymbol{q} \cdot \boldsymbol{x}}$

which have significance of complex envelopes for wavetrains, and using a band narrowness of the wave packets, we obtain

$H=\frac{i}{2} \sum_{n=1}^{3} \boldsymbol{v}_{n} \int d \boldsymbol{x} u_{n} \nabla u_{n}^{*}+V \int d \boldsymbol{x} u_{1}^{*} u_{2} u_{3}+$ c.c.,

where envelope velocities $\boldsymbol{v}_{n}$ and interaction coefficient $V$ which is a function of the wave-vectors $\boldsymbol{k}_{1}, \boldsymbol{k}_{2}, \boldsymbol{k}_{3}$, are expressed as

$$
\begin{aligned}
\boldsymbol{v}_{n} & =\partial \omega_{\boldsymbol{k}_{n}} / \partial \boldsymbol{k}_{n} \\
& =\left(\boldsymbol{k}_{n}^{2}+1\right)^{-2}\left\{\kappa_{n}^{2}-\sigma_{n}^{2}-1,2 \kappa_{n} \sigma_{n}\right\}, \\
V & =V_{\boldsymbol{k}_{1}, \boldsymbol{k}_{2}, \boldsymbol{k}_{3} .}
\end{aligned}
$$


Now, let us consider interaction between two Rossby-like wave packets $\boldsymbol{k}, \boldsymbol{k}_{1}$ and the null mode packet $\boldsymbol{k}_{2}$. So far as in this case $\kappa_{1}, \kappa_{2}<0$ but $\kappa_{3}>0$, from Eq. (68) we have

$V=-\frac{i\left(\sigma_{2} \kappa_{3}-\kappa_{2} \sigma_{3}\right)^{2}}{2 \pi \sqrt{\left|\kappa_{1} \kappa_{2} \kappa_{3}\right|}}\left(\frac{\partial \omega_{\boldsymbol{k}_{1}}}{\partial \sigma_{1}}-\frac{\partial \omega_{\boldsymbol{k}_{2}}}{\partial \sigma_{2}}\right)$.

The space-time evolution of the packets can be described from equations

$\partial_{t} u_{j}=-i \frac{\delta H}{\delta u_{j}^{*}}$,

which take the form

$\partial_{t} u_{1}+v_{1} \nabla u_{1}=-i V u_{2} u_{3}$,

$\partial_{t} u_{2}+\boldsymbol{v}_{2} \nabla u_{2}=-i V^{*} u_{1} u_{3}^{*}$,

$\partial_{t} u_{3}=-i V^{*} u_{2}^{*} u_{1}$.

\section{Null-mode mechanism of flow generation}

As shown by Longuet-Higgins and Gill (1969), the mechanism of a direct triad resonance which takes into account only Rossby-like modes, cannot be responsible for exciting zonal flows if weak corrections for sideband resonance are ignored. This conclusion is completely confirmed by our results and is readily apparent from Eq. (68) whereby coupling coefficient $V_{\boldsymbol{k}_{1}, \boldsymbol{k}_{2}, \boldsymbol{k}_{3}}$ vanishes as $\boldsymbol{k}_{1}=\boldsymbol{k}_{2}$.

As will be shown later, in contrast to the cited works, our approach which uses the null-mode concept admits the generation of zonal flows due to direct triad resonance Eq. (69) involving two Rossby-like wave packets $\boldsymbol{k}_{1}, \boldsymbol{k}_{2}$ and a packet of null mode $\boldsymbol{k}_{3}$. These resonances are governed by the system of three nonlinear Eqs. (69) which in general can be solved by inverse scattering method when initial envelopes are non-overlapping. Using this technique, Zakharov (1976) obtained special solution describing a physically important effect - a parametric decay of a pump wave into secondary waves.

\subsection{Noncoplanar case $\boldsymbol{v}_{1} \times \boldsymbol{v}_{2} \neq 0$}

Consider $u_{1}, u_{2}$ as envelopes of Rossby-like waves fixed in the initial stage at $t \rightarrow-\infty$ when the null-mode packet $u_{3}$ is absent. Then, using characteristic coordinates $\tau_{1}, \tau_{2}, \tau_{3}$ defined as

$\boldsymbol{x}=-\boldsymbol{v}_{1} \tau_{1}-\boldsymbol{v}_{2} \tau_{2}, \quad t=-\tau_{1}-\tau_{2}-\tau_{3}$,

Zakharov's solution can be presented in the form

$$
\begin{aligned}
& u_{1}=\frac{1}{V} \frac{f_{2} f_{3}}{1+\left(F_{1}+F_{2}\right) F_{3}}, \\
& u_{2}=\frac{1}{V} \frac{f_{1} f_{3}}{1+\left(F_{1}+F_{2}\right) F_{3}}, \\
& u_{3}=-\frac{i}{V} \frac{f_{1}^{*} f_{2} F_{3}}{1+\left(F_{1}+F_{2}\right) F_{3}},
\end{aligned}
$$

where $f_{i}=f_{i}\left(\tau_{i}\right)$ are arbitrary, square-integrable, complex functions, and $F_{i}$ are determined by

$$
\begin{aligned}
& F_{1}=\int_{\tau_{1}}^{+\infty} d s\left|f_{1}\right|^{2}, \quad F_{2}=\int_{-\infty}^{\tau_{2}} d s\left|f_{2}\right|^{2}, \\
& F_{3}=\int_{\tau_{3}}^{+\infty} d s\left|f_{3}\right|^{2} .
\end{aligned}
$$

To interpret correctly the initial-value problem in terms of characteristic coordinates $\tau_{1}, \tau_{2}, \tau_{3}$, we note that due to the minus signs in Eq. (75), fixing any two of the characteristic coordinates would send the third characteristic coordinate to $+\infty$ if $t \rightarrow-\infty$, or to $-\infty$ if $t \rightarrow+\infty$. Thus, the initial states will be located where any one of the characteristic coordinates approaches $+\infty$, and the final states will be located where any one of the characteristic coordinates approaches $+\infty$. Therefore, quantities

$u_{i}^{+}=\lim _{\tau_{i} \rightarrow+\infty} u_{i}, \quad u_{i}^{-}=\lim _{\tau_{i} \rightarrow-\infty} u_{i}$,

correspond to the initial and final profiles, respectively.

Considering the integral characteristic

$I_{i}^{+}=\int d \boldsymbol{x}\left|u_{i}^{+}\right|^{2}, \quad I_{i}^{-}=\int d \boldsymbol{x}\left|u_{i}^{-}\right|^{2}$

as initial and final intensities of the packets, we can obtain a convenient formula for their calculation

$$
\begin{aligned}
I_{i}^{ \pm} & =s_{i} \frac{g^{1 / 2}}{V} \\
& \times \int d \tau_{j} d \tau_{k} \partial_{j} \partial_{k} \ln \left(1+\left.\left(F_{1}+F_{2}\right) F_{3}\right|_{\tau_{i}= \pm \infty}\right),
\end{aligned}
$$

where $s_{i}=\operatorname{sign} \partial_{i} F_{i}$ and $g^{1 / 2}=\sqrt{\boldsymbol{v}_{1}^{2} \boldsymbol{v}_{2}^{2}-\left(\boldsymbol{v}_{1} \boldsymbol{v}_{2}\right)^{2}}$ is the Jacobian of transformation Eq. (75).

Denoting

$$
Y_{i}=\int_{-\infty}^{+\infty} d s\left|f_{i}\right|^{2}, \quad i=1,2,3,
$$

we can compute the initial and final integral intensities of the wave packets.

As shown by Zakharov (1976), in the initial stage when the wave packet $u_{3}$ is absent, the integral intensities of $u_{1}$, $u_{2}$, and $u_{3}$ are determined as

$$
\begin{aligned}
I_{1}^{+} & =-\frac{g^{1 / 2}}{V} \int d \tau_{2} d \tau_{3} \partial_{2} \partial_{3} \ln \left(1+F_{2} F_{3}\right) \\
& =\frac{g^{1 / 2}}{V} \ln \left(1+Y_{2} Y_{3}\right) \\
I_{2}^{+} & =\frac{g^{1 / 2}}{V} \int d \tau_{2} d \tau_{3} \partial_{1} \partial_{3} \ln \left(1+\left(F_{1}+Y_{2}\right) F_{3}\right) \\
& =\frac{g^{1 / 2}}{V} \ln \left(1+\frac{Y_{1} Y_{3}}{1+Y_{2} Y_{3}}\right) \\
I_{3}^{+} & =0 .
\end{aligned}
$$


In final stage we have all three packets with intensities

$$
\begin{aligned}
I_{1}^{-} & =-\frac{g^{1 / 2}}{V} \int d \tau_{2} d \tau_{3} \partial_{2} \partial_{3} \ln \left(1+\left(Y_{1}+F_{2}\right) F_{3}\right) \\
& =\frac{g^{1 / 2}}{V} \ln \left(1+\frac{Y_{2} Y_{3}}{1+Y_{1} Y_{3}}\right), \\
I_{2}^{-} & =\frac{g^{1 / 2}}{V} \int d \tau_{2} d \tau_{3} \partial_{1} \partial_{3} \ln \left(1+F_{1} F_{3}\right) \\
& =\frac{g^{1 / 2}}{V} \ln \left(1+Y_{1} Y_{3}\right), \\
I_{3}^{-} & =-\frac{g^{1 / 2}}{V} \int d \tau_{2} d \tau_{3} \partial_{1} \partial_{2} \ln \left(1+\left(F_{1}+F_{2}\right) Y_{3}\right) \\
& =\frac{g^{1 / 2}}{V} \ln \left(1+\frac{Y_{3}^{2} Y_{1} Y_{2}}{1+Y_{3}\left(Y_{1}+Y_{2}\right)}\right) .
\end{aligned}
$$

It is convenient to introduce the renormalized intensities

$J_{i}^{ \pm}=g^{-1 / 2} V I_{i}^{ \pm}$

Then, eliminating quantities $Y_{1}, Y_{2}, Y_{3}$ from Eqs. (82-87), we can express final intensities $J_{i}^{-}(i=1,2,3)$ in terms of the initial ones as

$J_{1}^{-}=J_{1}^{+}+J_{2}^{+}-\ln \left[1+e^{J_{1}^{+}}\left(e^{J_{2}^{+}}-1\right)\right]$,

$J_{2}^{-}=\ln \left[1+e^{J_{1}^{+}}\left(e^{J_{2}^{+}}-1\right)\right]$,

$J_{3}^{-}=-J_{2}^{+}+\ln \left[1+e^{J_{1}^{+}}\left(e^{J_{2}^{+}}-1\right)\right]$.

\subsection{Coplanar case}

In a coplanar case when $\boldsymbol{v}_{1} \times \boldsymbol{v}_{2}=0$, Eqs. (74) become

$$
\begin{aligned}
\dot{u}_{1}+v_{1} \partial u_{1} / \partial \xi & =-i V u_{2} u_{3}, \\
\dot{u}_{2}+v_{2} \partial u_{2} / \partial \xi & =-i V^{*} u_{1} u_{3}^{*}, \\
\dot{u}_{3} & =-i V^{*} u_{1} u_{2}^{*} .
\end{aligned}
$$

and describe the evolution in time and one spatial dimension of the three-wave resonant interaction. The spatial variable $\xi$ used as a coordinate along the line of propagation $\boldsymbol{v}_{1}$, is related to $\boldsymbol{x}$ by expression

$\xi=x \cos \varphi+y \sin \varphi$,

As shown in Fig. 2, angle $\varphi$ defines the direction of the propagation $(-\pi / 2<\varphi<\pi / 2)$. Together with frequency $\omega_{1}$, it can be used for parameterization of the resonant triplet

$\boldsymbol{k}_{3}=2 r(\cos \varphi, \sin \varphi)$,

$\boldsymbol{k}_{1,2}=-\frac{1}{2 \omega_{1}}(1,0) \pm \frac{1}{2} \boldsymbol{k}_{3}$,

where $r=\sqrt{\omega_{1}^{-2} / 4-1}$.

We consider the situation when in the initial stage at $t \rightarrow-\infty$, Rossby-like wave packets $u_{1}, u_{2}$ are localized at infinities $(\xi= \pm \infty)$ and the null-mode packet $u_{3}$ is absent. Velocities of the wave packets can be evaluated from relations

$v_{1,2}=\left|\partial \omega_{\boldsymbol{k}} / \partial \boldsymbol{k}\right|_{\boldsymbol{k}=\boldsymbol{k}_{1,2}}=\frac{4 \omega_{1}^{3} r}{2 \omega_{1} r \cos \varphi \mp 1}$. which show that $v_{1}<0$ and $v_{2}>0$. Thus, these packets move towards each other and after some time collide generating null-mode packet $u_{3}$. At the final stage at $t \rightarrow \infty$, when Rossby-like wave packets run away into infinities, all that remains in the interaction region is the immovable null-mode packet $u_{3}$ which will never leave the place of its creation. This situation corresponds to the special solution

$$
\begin{aligned}
u_{1}= & -\frac{2 p_{1} \sqrt{-v_{1}\left(v_{2}-v_{1}\right)}}{V D} \\
& \times\left(e^{\eta_{2}}+\frac{p_{1} v_{1}-p_{2} v_{2}}{p_{1} v_{1}+p_{2} v_{2}} e^{-\eta_{2}}\right), \\
u_{2}= & -\frac{2 p_{2} \sqrt{v_{2}\left(v_{2}-v_{1}\right)}}{V D} \\
& \times\left(e^{\eta_{1}}-\frac{p_{1} v_{1}-p_{2} v_{2}}{p_{1} v_{1}+p_{2} v_{2}} e^{-\eta_{1}}\right), \\
u_{3}= & -i \frac{4 p_{1} p_{2}\left(v_{2}-v_{1}\right) \sqrt{-v_{1} v_{2}}}{V\left(p_{1} v_{1}+p_{2} v_{2}\right) D},
\end{aligned}
$$

where

$$
\begin{aligned}
\eta_{1} & =p_{1}\left(\xi-v_{1} t-\xi_{1}\right) \\
\eta_{2}= & p_{2}\left(\xi-v_{2} t-\xi_{2}\right) \\
D= & \left(e^{\eta_{1}}+e^{-\eta_{1}}\right)\left(e^{\eta_{2}}+e^{-\eta_{2}}\right) \\
& -\frac{4 p_{1} p_{2} v_{2} v_{1}}{\left(p_{1} v_{1}+p_{2} v_{2}\right)^{2}} e^{-\eta_{1}-\eta_{2}}
\end{aligned}
$$

Solutions of this sort were first considered by Zakharov and Manakov (1973). According to Eqs. (99) and (100), wave packets $u_{1}, u_{2}$ are characterized by arbitrary amplitudes $b_{2}$, but the widths of the packets are related. From Eqs. (102) and (103) it follows that if $l$ is the width of packet $u_{1}$, the width of packet $u_{2}$ is $v_{1} / v_{2}$ times smaller, where in accordance with Eq. (98), $v_{1} / v_{2} \geq 1$.

\section{Estimates and conclusion}

After the two original wave packets, $u_{1}$ and $u_{2}$, beat against each other for some time, they escape from the interaction region leaving behind the null-mode packet, $u_{3}$. Thus, at the final stage, in accordance with Eq. (76), we have the residual field

$$
\bar{a}=u_{3}^{-}=-\frac{i}{V} \frac{f_{1}^{*} f_{2} Y_{3}}{1+\left(F_{1}+F_{2}\right) Y_{3}} .
$$

Because this disturbance is immovable, it will never leave the place of its creation.

Using Eq. (76) and assuming that $\bar{a}$ is a slow variable, i.e. $\partial \bar{a} / \partial x=\partial \bar{a} / \partial y=0$, we can compute residual fields. At first, from Eqs. (60) and (61), we obtain

$$
\begin{aligned}
& q=\sigma_{3}\left|\kappa_{3}\right|^{-1 / 2} \bar{a} \exp \left(i \boldsymbol{k}_{3} \boldsymbol{x}\right)+c . c ., \\
& p=-\kappa_{3}\left|\kappa_{3}\right|^{-1 / 2}\left(y+\frac{i}{\sigma_{3}}\right) \bar{a} \exp \left(i \boldsymbol{k}_{3} \boldsymbol{x}\right)+c . c .
\end{aligned}
$$


Next, from Eq. (17) we find the stream-function

$\psi=\bar{\psi} \exp \left(2 i \boldsymbol{k}_{3} \boldsymbol{x}\right)+$ c.c.,

where its envelope $\bar{\psi}$ is expressed as

$\bar{\psi}=\frac{i \sigma_{3}\left|\kappa_{3}\right| \bar{a}^{2}}{4 \boldsymbol{k}_{3}^{2}+1}$.

To gain greater insight into the physical significance of the results, we make some numerical estimates for an ocean model. In geostrophic approximation, the basic parameters are defined as $a=\sqrt{g h} / f, \beta=\partial_{2} f$, where $f, g$ and $h$ denote the Coriolis force, the acceleration of gravity and the mean depth of layer. Choosing $a=50 \mathrm{~km}$ (baroclinic Rossby radius), we consider middle latitudes where $\beta=10^{-13} \mathrm{~cm}^{-1} \mathrm{~s}^{-1}$. Then, in accordance with Eq. (5), we find the scales of length $L$ and time $T$ under which model parameters $a$ an $\beta$ become unity:

$L=a=50 \mathrm{~km}, \quad T=(\beta a)^{-1}=2 \cdot 10^{6} \mathrm{~s}$.

Let us suppose that in the initial state two Rossbywave packets with wave vectors $\boldsymbol{k}_{1}=\{-1.59,2.45\}$ and $\boldsymbol{k}_{2}=\{-5.45,1.41\}$ move with velocities $\boldsymbol{v}_{1}=\{-0.049,-0.086\} \quad$ and $\boldsymbol{v}_{2}=\{0.025,-0.014\}$, and have $\psi_{1}^{0}=1.2 \cdot 10^{-2}$ and $\psi_{2}^{0}=2.6 \cdot 10^{-5}$, respectively. As numerical simulation shows, these packets generate a null-mode packet with $\boldsymbol{k}_{3}=\{3.86,1.03\}$ and $\psi_{3}^{0}=2.3 \cdot 10^{-4}$.

Let us conclude with a few remarks. In this paper, we have considered the simplest model which possesses both strong dispersion and strong inhomogeneity and nonlinearity. However, this model has permitted us to show how the null-mode concept changes traditional ideas about the influence of nonlinear interactions. We have also discussed the basis for the proposed approach and highlighted important non-trivial generalization for the operator expressions of normal variables.

The choice of the method is motivated by several reasons. Successful solution of a problem of theoretical physics often depends on which descriptive formalism, i.e. the mathematical framework, is chosen (see Zakharov, 1985). In fact, there may exist several analytical approaches which under consistent application lead to the same final result. Theoreticians, however, often tend to be biased in favor of one while instinctively resisting attempts aiming to explore others: they claim that new approaches do not contribute anything new. Consequently, not all possible analytical frameworks are treated equally and some get pushed out by the others. As an example, in medieval times European universities developed several coexisted algorithms for arithmetic division, but all of them except for the single one are obsolete nowadays. However, the prevailing method should not be the one that is most habitual. The "best" scheme should be the one that is most adequate for the problem in question. In fact, after the period of implementation and adaptation, the framework itself may start affecting the style of thinking and enriching scientific language. Finally, it may begin to define the way in which new physical problems are stated. This happened, for instance, with the Feynmann diagram technique which originally seemed to be merely a simplification method in the perturbation theory.

The analogous situation has happened with the HA which is based on the fundamental fact that governing equations of a hydrodynamical system possess a hidden Hamiltonian structure.

\section{Appendix A Hamilton approach}

There exist different methods which use the adjective "Hamiltonian", and there are numerous papers with titles where the adjective "Hamiltonian" is used. That is why, when talking about the Hamiltonian method, it is necessary to define more precisely which one of the versions is implied. The so-called "Hamiltonian principle" and the so-called "Hamiltonian description" can mean different approaches.

The point of the departure for one of the approaches is the integral (action) of a hydrodynamical system taken in the form

$$
\begin{aligned}
S & =\int d t \mathcal{L}\left[u_{i}, \partial_{t} u_{i}\right] \\
& \equiv \int d t\left\{\left(\int d \boldsymbol{x} \hat{A}_{j}\left[u ; \boldsymbol{x}, \boldsymbol{x}_{1}\right] \partial_{t} u_{j}\left(\boldsymbol{x}_{1}\right)\right)-H[u]\right\} .
\end{aligned}
$$

Here, $\mathcal{L}$ is the Lagrangian of the hydrodynamical system, $H$ is the Hamiltonian of the same system, $\partial_{t} u_{k}$ is the partial derivative of a field variable with respect to time. Variations of the action, $S$, with respect to hydrodynamical field variables $u=\left(u_{k}\right)$ lead to the evolution equation $\delta S=0$, which is equivalent to

$$
\int d \boldsymbol{x}_{1} \hat{\omega}_{i k}\left[u ; \boldsymbol{x}, \boldsymbol{x}_{1}\right] \partial_{t} u_{k}\left(\boldsymbol{x}_{1}\right)=\frac{\delta H}{\delta u_{i}(\boldsymbol{x})} .
$$

Here, $\delta / \delta u_{m}$ are the operator of functional derivation, $\omega_{i k}$ is the symplectic form defined by the condition $\hat{\omega}_{i k}\left[\boldsymbol{x}, \boldsymbol{x}_{1}\right]=\delta \hat{A}_{i}\left[u\left(\boldsymbol{x}_{1}\right)\right] / \delta u_{k}(\boldsymbol{x})-\delta \hat{A}_{k}[u(\boldsymbol{x})] / \delta u_{i}\left(\boldsymbol{x}_{1}\right)$.

The approach proceeding from the extremum of Eq. (A1) and based on the use of equations Eq. (A2) has a wide dissemination. Reviews on applications of the variational principle of least action with a hydrodynamical Lagrangian density, can be found in works of Bretherton (1970); Henyey (1983); Salmon (1988) (see also publications relevant to this aspect in Abarbanel et al. (1986); Holm et al. (1985) and references therein; in context of the Hamiltonian formulation of Rossby wave model see for instance Lynch (2002)).

The other version of the Hamiltonian description proceeds from the evolution equations in the form

$$
\begin{aligned}
\partial_{t} u_{i}(\boldsymbol{x}) & =\left\{u_{i}, H\right\} \\
& \equiv \int d \boldsymbol{x}_{1}\left\{u_{i}(\boldsymbol{x}), u_{j}\left(\boldsymbol{x}_{1}\right)\right\} \frac{\delta H}{\delta u_{j}\left(\boldsymbol{x}_{1}\right)} .
\end{aligned}
$$

Here, $\left\{u_{i}(\boldsymbol{x}), u_{j}\left(\boldsymbol{x}_{1}\right)\right\}$ is the functional Poisson bracket, $H$ is the Hamiltonian of the hydrodynamical system (generally speaking, the full energy of a fluid). This latter form 
seems to be preferable sometimes from the physical point of view since it arises naturally in many known hydrodynamical models.

A particular case, the canonical form of the Hamilton approach, is given by the set of equations in terms of functional derivatives

$\partial_{t} q_{i}=\frac{\delta H}{\delta p_{i}}, \quad \partial_{t} p_{i}=-\frac{\delta H}{\delta q_{i}}$,

where $q_{i}, p_{i}, i=1,2, \ldots N$ are canonical variables, and $H$ is the Hamiltonian of the system.

Equations (A2) and (A3) would become equivalent if there existed a one-to-one transformation, i.e. if there existed relation

$$
\begin{aligned}
& \int d \boldsymbol{x}_{2} \hat{\omega}_{i j}\left[u ; \boldsymbol{x}_{1}, \boldsymbol{x}_{2}\right]\left\{u_{j}\left(\boldsymbol{x}_{2}\right), u_{k}\left(\boldsymbol{x}_{3}\right)\right\} \\
& =\delta_{i k} \delta\left(\boldsymbol{x}_{1}-\boldsymbol{x}_{3}\right) .
\end{aligned}
$$

Such a scenario is realized when the functional Poisson brackets are non-degenerated. In this case, it would be absolutely irrelevant which of the formulations was taken as a point of departure.

However, for systems with degenerated functional Poisson brackets $\left\{u_{j}, u_{k}^{\prime}\right\}$, which admit solutions of $\left\{u_{j}, C_{k}\right\}=0$ for Casimirs $C_{k}$, transformations Eq. (A5) are impossible. Such a situation is observed for hydrodynamical models (see, for example, Arnold, 1978).

If from the beginning one is forced to work within the class of models determined by evolution Eqs. (A2), it is necessary to go through the process not only of searching for the canonical variables, but also ascertaining their connection with physically-observed field quantities (for example, one needs to elucidate the sense of multi-valued Clebsch representations), then invent models of hydrodynamic systems with unusual properties, and so on.

Even if the necessary structure of the Lagrangian is guessed or selected in some intuitive way, the use of the variational principle Eq. (A1) requires the formulation of additional postulates concerning latent constraints, the physical interpretation of which is not always obvious.

In the historical context, the Hamiltonian description in the forme of Eq. (A3) is directly driven from physically-based presumptions about the type of evolution of hydrodynamical systems and their internal properties (see Goncharov and Pavlov, 1997).

The Hamiltonian approach Eq. (A3) was initially developed in fluid dynamics mainly for pragmatic goals, as a method of solving some concrete hydrodynamical problems. For example, it appears to be very effective in finding nonlinear evolution equations for interacting waves (for details, see Zakharov et al., 1985). It was immediately noticed that the Hamiltonian method possesses a number of advantages in comparison with traditional approaches. In particular, a) the Hamiltonian approach is not tied to a particular choice of "field coordinates". Specific features of a medium turn out to be unessential to a large extent; b) many versions of the perturbation theory may be simplified and standardized; c) the method is rather economical because an asymptotic expansion can be made in the beginning; d) the physical meaning of the results of calculations obtained for a particular system can be easily revealed. In early 1970s, (see work Zakharov and Faddeev, 1971), it became apparent that the majority of nonlinear evolution equations integrated by the inverse scattering method possess a Hamiltonian structure, i.e. they are the infinite-dimensional analogues of the Hamilton equations of classical mechanics.

Approximately at the same time the general physical essence of the Hamiltonian approach was realized. It became clear that in classical physics many of the conservative models which use a field concept, possess a hidden Hamiltonian structure. Many hydrodynamical models happened to be among them.

The construction and successful use of canonical variables for studying surface gravity waves Zakharov (1968) presented one of the most impressive examples of the application of the Hamiltonian approach in hydrodynamics and gave an impetus to elaborate the general wave theory for nonlinear, dispersive media in the framework of the canonical Hamiltonian formalism. The issue of determining canonical variables was essential to the development of the Hamiltonian method. For many fluid dynamic systems, the canonical variables in Clebsch representation have been introduced in an intuitive way (examples are given in Seliger and Whitham, 1968; Zakharov and Kuznezov, 1997). There even exists an opinion that the canonical variables may only be guessed (see L'vov, 1994). In reality, it is not correct, there exists a regular procedure for finding canonical variables (see Goncharov and Pavlov, 1993, 1997).

If the Hamiltonian approach Eq. (A3) merely offered a new vision of familiar results, it would deserve little attention. However, enough evidence has accumulated that the Hamiltonian approach, together with the methods of modern classical mechanics (see Dubrovin and Novikov, 1989; Arnold, 1978), comprise a powerful tool for fluid dynamics research. Conservation laws, stability conditions, asymptotic approximations and useful variable transformations, all acquire logical motivation and transparency that is often lacking when the corresponding manipulations are applied directly to the traditional evolution equations.

Acknowledgements. This work was partly supported by the Russian Foundation for Basic Research (grant No.00-05-64019-a). The authors thank A. Dyment and E. P. Tito for useful remarks.

Edited by: A. R. Osborne

Reviewed by: two referees

\section{References}

Abarbanel, H. D. I., Holm, D. D., Marsden, J. E., and Ratiu, T. S.: Nonlinear stability analyzis of stratified fluid equilibria, Phyl. Trans. Roy. Soc., London A 318, 349-409, 1986.

Arnold, V. I.: Mathematical Methods of Classical Mechanics, Springer-Verlag, New York, 1978. 
Bogoljubov, N. N. and Shirkov, D. B.: Introduction to the Theory of Quantized Fields, 3rd Ed., Interscience, 1959; Wiley, 1980.

Bretherton, F. P.: A note on Hamilton's principle for perfect fluids, J. Fluid Mech., 44(1), 19-31, 1970.

Dirac, P. A. M.: Generalized Hamiltonian dynamics, Proc. Roy. Soc., A 246, 326-332, 1958.

Dubrovin, B. A. and Novikov, S. P.: Hydrodynamics of weekly deformed soliton latices: Differential geometry and Hamiltonian theory, Russ. Math. Surveys., 44 (6), 35-124, 1989.

Goncharov, V. P.: Hamiltonian representation of the equations of hydrodynamics and its use for describing wave motions in shear flows, Izvestiya Acad. Sci. USSR, Atmos. Ocean Phys., 20 (2), 92-99, 1984.

Goncharov, V. P. and Pavlov, V. I.: Problems of hydrodynamics in Hamiltonian description, Moscow University Press, Moscow, 1993.

Goncharov, V. P. and Pavlov, V. I.: Some remarks on the physical fondation of the Hamiltonian description of fluid motions, Eur. J. Mech. B/Fluids, 16 4, 509-555, 1997.

Goncharov, V. P. and Pavlov, V. I.: On the Hamiltonian approach: Applications to geophysical flows, Nonl. Proc. in Geoph., 1, 219-240, 1998.

Goncharov, V. P. and Pavlov, V. I.: Large-scale vortex structures in shear flows, Eur. J. Mech. B/Fluids, 19, 831-854, 2000.

Goncharov, V. P. and Pavlov, V. I.: Multipetal vortex structures in two-dimensional models of geophysical fluid dynamics and plasma, Journ. Exper. and Theor. Physics, 119 (4), 685-699, 2001.

Goncharov, V. P., Gryanik, V. M. and Pavlov, V. I.: Venusian "hot spot": physical phenomenon and its quantifiation, Phys. Rev., E 66, 066304-1-066303-11, 2002.

Gruzinov, A. V.: Contour dynamics of the Hasegawa-Mima equation, JETP Lett., 55, 1, 75-78, 1992.

Hasegawa, A. and Mima, K.: Stationary spectrum of strong turbulence in magnetized nonuniform plasma, Phys. Rev. Lett., 39, 205-208, 1977.

Henyey, F. S.: Hamiltonian description of stratified fluid dynamics, Phys. Fluid, 26 (1), 40-47, 1983

Holm, D. D., Marshden, J. E., Ratiu, T., and Weinstein, A.: Nonlinear stability of fluid and plasma equilibria, Phys. Rep., 123, 1-116, 1985.

Kadomtsev, B. B.: Plasma turbulence, Academic, New York, 1965.

Krall, N. A. and Trivelpiece, A. W.: Principles of Plasma Physics, McGrew-Hill Book Company, 1973.
Lamb, H.: Hydrodynamics, Cambridge University Press, Cambridge, 1932

Longuet-Higgins, M. S. and Gill, A. E.: Proc. Roy. Soc., A 299, $120,1969$.

Lundgreen, T. S.: Hamilton's variational principle for perfectly conducting plasma continuum, Phys. Fluid, 6 (7), 898-904, 1963.

L'vov, V. S.: Wave Turbulence Under Parametric Excitation, Springer Series in Nonlinear Dynamics, Springer-Verlag Berlin Heidelberg, 1994.

Lynch, P.: Hamiltonian methods for geophysical fluid dynamics: An introduction, IMA, Univ. of Minnesota, Preprints 1838, 1 32, 2002.

Pavlov, V. I., Buisine, D., and Goncharov, V. P.: Formation of vortex clusters on a sphere, Nonl. Proc. in Geoph., 8, 9-19, 2001.

Pedlosky, J.: Geophysical fluid dynamics, 2nd Ed., Springer-Verlag, New York, 1986.

Petviashvili, V. I. and Pohotelov, O. A.: Solitary waves in plasma and atmosphere, Energoatomizdat, Moscow, 1989.

Salmon, R.: Hamiltonian fluid mechanics, Ann. Rev. Fluid Mech., 20, 225-256, 1988.

Seliger, R. L. and Whitham, G. B.: Variational principles in continuum mechanics, Proc. R. Soc. London, A 305, 1-25, 1968.

Similon, P. L. and Sudan, R. N.: Plasma turbulence, Annu. Rev. Fluid Mech., 22, 317, 1990.

Zakharov, V. E.: Stability of periodic waves of finite amplitude of the surface of a deep fluid, J. Appl. Mech. Tech. Phys., 2, 190 194, 1968.

Zakharov, V. E.: Exact solutions to the problem of the parametric interaction of three-dimensional wave packets, Sov. Phys. Dokl., 21, 322, 1976 .

Zakharov, V. E. and Faddeev, L. D.: The Korteweg-de Vries equation as Hamiltonian system, Func. An. and Its Appl., 5, 4, 18-27, 1971.

Zakharov, V. E. and Kuznetsov, E. A.: Hamilton formalizm for systems of the hydrodynamic type, edited by Novikov, S. P., Sov. Sci. Rev., 91, 1310, 1986.

Zakharov, V. and Kuznezov, E.: Hamiltonian formalism for nonlinear waves, Physics-Uspekhi, 40 (11), 1137-1167, 1997.

Zakharov, V. E. and Manakov, S. V.: On resonant interaction of wave packets in non-linear media, JETP Lett., 18, 243-247, 1973.

Zakharov, V. E., Musher, S. L., and Rubenchik, A. M.: Hamiltonian approach to the description of nonlinear plasma phenomena, Phys. Rep., 129 (5), 287-366, 1985. 